\title{
Iniciação científica, vivências acadêmicas e rendimento de graduandos em Odontologia e Medicina
}

\author{
Clara Letícia da Costa Carvalho*; Gabriela Victorelli**; Rui Barbosa de Brito Júnior***; Almenara de \\ Souza Fonseca Silva****; Luciane Zanin***; Flávia Martão Flório****
}

\author{
* Graduanda em Odontologia, Faculdade São Leopoldo \\ Mandic \\ ** Doutoranda em Ensino em Saúde, Faculdade de Ciências \\ Médicas, Universidade de Campinas \\ *** Professor (a), cursos de Odontologia e Medicina, Faculdade \\ São Leopoldo Mandic \\ **** Professora, curso de Odontologia, Faculdade São Leopoldo \\ Mandic \\ Recebido em 11/02/2019. Aprovado em 27/12/2019.
}

\begin{abstract}
RESUMO
O Programa de Iniciação Cientifica (PIC) busca despertar a vocação e estimular o desenvolvimento do pensamento científico em graduandos, tendo sido identificado previamente que a participação no PIC institucional apresentou impacto positivo no coeficiente de rendimento (CR) de estudantes de Odontologia, o que não ocorreu com os de Medicina. Desta forma, desenvolveu-se o presente estudo observacional, que visa avaliar aspectos pessoais, contextuais e vocacionais das vivências acadêmicas que possam ter influenciado este achado. Participaram 123 alunos PIC, sendo $74(60,2 \%)$ do curso de Odontologia (O) e 49 (39,9\%) do curso de Medicina (M). A versão reduzida do Questionário de Vivências Acadêmicas (QVA-r com 54 questões e resposta em escala do tipo Likert) foi aplicada. Os dados foram tabulados e analisados utilizando-se o teste $t$ de Student $(\alpha=5 \%)$. Os principais resultados apontaram que as dimensões carreira, estudo e institucional diferiram entre os grupos, com maiores pontuações médias para estudantes do curso de Medicina na dimensão carreira $(M=4,1 \pm 0,6 ; \mathrm{O}=3,8 \pm 0,4)$. Para os do curso de Odontologia, maiores pontuações médias foram observadas nas dimensões estudo $(\mathrm{M}=3,6 \pm 0,5 ; \mathrm{O}=3,9 \pm 0,7)$ e institucional $(\mathrm{M}=3,7 \pm 0,2 ; \mathrm{O}=4,1 \pm 0,2)$. Não houve diferença entre os cursos considerando as dimensões pessoal e interpessoal e o QVAr geral. Conclui-se que vivências acadêmicas diferenciadas podem ter influenciado o antagônico impacto do PIC no CR dos estudantes de Odontologia e Medicina.
\end{abstract}

Descritores: Pesquisa Científica. Rendimento Escolar. Formação Profissional. 


\section{INTRODUÇÃ̃O}

O reconhecimento da importância estratégica da ciência e a necessidade de institucionalizar as ações de incentivo e fomento culminaram com a criação, em 1951, do Conselho Nacional de Desenvolvimento Científico e Tecnológico (CNPq), tendo entre suas atribuições a promoção e o estímulo ao desenvolvimento da investigação científica e tecnológica em qualquer domínio do conhecimento ${ }^{1}$. Desde então, favoreceu-se no Brasil o desenvolvimento da investigação científica e tecnológica, com a regularização e o fomento de pesquisas realizadas durante os cursos de graduação, com o objetivo de associar o ensino à pesquisa além de estimular a prática da iniciação científica² ${ }^{2}$.

A iniciação cientifica é considerada um método de ensino e aprendizagem que não se limita à mera introdução do estudante na área científica, já que o expõe a situações de aprendizagem que vão além daquelas encontradas em sala de aula, visando despertar a vocação científica, incentivando talentos potenciais entre estudantes de graduação ${ }^{3}$. Estudo anterior do tipo caso controle realizado na instituição de ensino superior (IES) do presente estudo buscou investigar o impacto da IC no desempenho dos estudantes de seus dois cursos de graduação, Odontologia e Medicina, e identificou que o coeficiente de rendimento (CR) dos graduandos vinculados ao PIC foi sempre maior do que o dos não participantes do programa. No entanto, considerandose apenas os alunos PIC, verificou-se que após a inclusão no programa houve aumento no $\mathrm{CR}$ dos acadêmicos do curso de Odontologia ${ }^{4}$, enquanto no curso de Medicina houve diminuição significativa ${ }^{5}, \mathrm{o}$ que motivou buscar compreender as razões dessa diferença.

Diversos aspectos vêm sendo estudados na literatura no sentido de compreender os fatores que podem influenciar no rendimento acadêmico dos estudantes. Cunha e Carrillo (2005) $)^{6}$ discutem que as dificuldades da integração do aluno ao contexto universitário passam por questões pessoais e também decorrem das novas exigências acadêmicas e do novo ambiente, explicitadas por Soares et al. (2014) ${ }^{7}$ : maior autonomia e organização de seus horários, as aulas acontecem de maneira menos sequenciada do que na escola e os professores desempenham variadas funções. A diversidade das experiências no contexto universitário conduz a busca por mudanças tanto no âmbito pessoal, contextual e institucional, já que o aluno deve desenvolver a autonomia nos estudos e a instituição deve contribuir com o desenvolvimento de habilidades relacionais e cognitivas ${ }^{8}$.

Neste contexto, a compreensão de aspectos pessoais, contextuais e vocacionais das vivências acadêmicas ${ }^{9}$ é um ponto importante a ser considerado na compreensão desta temática ${ }^{10,11}$. O presente estudo avalia aspectos pessoais, interpessoais, de carreira, estudo e institucionais relacionados aos estudantes de Odontologia e Medicina de uma instituição privada de Campinas/SP), buscando compreender o impacto antagônico do PIC no CR dos acadêmicos.

\section{METODOLOGIA}

Este estudo do tipo observacional, transversal, de natureza quantitativa analítica foi conduzido de acordo com os preceitos determinados pela Resolução $\mathrm{n}^{\circ} 466$ de 2012, tendo sido aprovado pelo Comitê de Ética (CAAE 77378917.2.0000.5374 / Parecer: 2.345.870).

O período do estudo compreende as 5 primeiras edições do PIC (2013 a 2017), ao qual vincularam-se 161 estudantes, dentre os quais 92 do curso de Odontologia e 69 do curso de Medicina. Todos os acadêmicos vinculados ao PIC neste período foram contatados e convidados a participar do estudo.

Os estudantes foram abordados pessoalmente $\mathrm{e}$ para aqueles que não podiam fazer o preenchimento 
do questionário presencialmente, após muitas tentativas, enviou-se o instrumento em formulário eletrônico. Após 15 dias do envio do convite, foi realizado contato por Whatsapp, Facebook e e-mail para confirmação do recebimento, solicitação de resposta e reenvio do formulário eletrônico. Em caso de não efetivação do contato, foi feita nova abordagem pessoal para sanar dúvidas sobre a pesquisa e solicitar o preenchimento do instrumento.

Os 123 estudantes que aceitaram participar preencheram o Questionário de Vivências Acadêmicas - Versão Reduzida (QVA-r), adaptado à realidade do contexto brasileiro ${ }^{12}$. Este instrumento busca avaliar o modo como os estudantes percebem suas experiências acadêmicas na IES que frequentam. A versão do QVA-r utilizada é composta por 54 itens com respostas em escala do tipo Likert, variando de 1 (nada a ver comigo) a 5 (tudo a ver comigo), em 5 dimensões:

1. dimensão pessoal, que integra a avaliação do bemestar físico e psicológico, o seu equilíbrio emocional, a estabilidade afetiva e o otimismo;

2. dimensão interpessoal, que avalia as relações entre pares, as competências de relacionamento em situações de maior intimidade, o estabelecimento de amizades e a procura de ajuda;

3. dimensão carreira, que integra a avaliação de sentimentos relacionados ao compromisso com o curso, as perspectivas de carreira e os projetos vocacionais;

4. dimensão estudo, que inclui as competências de estudo, os hábitos de trabalho, a gestão do tempo, além da utilização da biblioteca e de outros recursos de aprendizagem; e

5. dimensão instituição, que integra a apreciação dos estudantes face à instituição de ensino frequentada, o desejo de permanecer ou mudar de instituição, o conhecimento e a apreciação da infraestrutura; também compreendida como o senso de pertencimento à IES.

Os dados coletados a partir dos questionários foram apurados por meio dos procedimentos estatísticos necessários, com o auxílio do software Bioestat 5.0. Para cada dimensão considerou-se a soma dos pontos e os valores intermediários (média, desvio padrão e mediana) de cada dimensão pela escala Likert, e do instrumento completo. O teste $\mathrm{t}$ de Student foi utilizado na análise das pontuações obtidas entre os cursos. Foram consideradas associações estatisticamente significantes aquelas que apresentaram valores de $\mathrm{p}$ iguais ou menores do que $5 \%(0,05)$.

\section{RESULTADOS}

A população era de 161 estudantes (92 do curso de Odontologia e 69 do curso de Medicina) (tabela 1) e amostra final contemplou 123 participantes, 60,2\% matriculados no curso de Odontologia e 39,9\% no curso de Medicina. A perda de indivíduos se deu pela ausência nos diversos momentos da aplicação do questionário (37 alunos) e pela recusa em participar da pesquisa (1 aluna). A taxa de resposta de $76,4 \%$ foi considerada satisfatória, considerando estudos de mesma natureza.

A tabela 2 apresenta os achados, considerando-se a média da soma dos pontos e a média da escala Likert respondida pelos grupos, para cada dimensão. Nota-se que as dimensões carreira, estudo e institucional diferiram entre os grupos, com maiores pontuações médias para os alunos do curso de Medicina na dimensão carreira e maiores para os estudantes do curso de Odontologia na dimensão estudo e institucional.

\section{DISCUSSÃO}

Os resultados obtidos apontam diferença nas vivências acadêmicas dos estudantes em função dos cursos de matrícula, no que tange às dimensões 
carreira, estudo e institucional. Nas dimensões pessoal e interpessoal não houve diferença.

Tabela 1. Frequência de participação dos alunos no Programa de Iniciação Cientifica em função do curso de matrícula e edições do programa

\begin{tabular}{ccccc}
\hline & \multicolumn{2}{c}{ Curso de Medicina } & \multicolumn{2}{c}{ Curso de Odontologia } \\
Edições PIC & $\mathbf{n}$ & $\mathbf{\%}$ & $\mathbf{n}$ & $\mathbf{\%}$ \\
\hline 1 $^{\text {a }}$ PIC 2013 & 2 & 2,9 & 9 & 9,8 \\
$2^{\mathrm{a}}$ - PIC 2014 & 7 & 10,2 & 19 & 20,6 \\
3 $^{\text {- PIC } 2015}$ & 6 & 8,7 & 16 & 17,4 \\
$4^{\text {a }}$ - PIC 2016 & 15 & 21,7 & 15 & 16,3 \\
$5^{\text {a }}$ - PIC 2017 & 39 & 56,5 & 33 & 35,9 \\
\hline Total & 69 & 100,0 & 92 & 100,0 \\
\hline
\end{tabular}

Tabela 2. Análise da soma dos pontos, média e mediana das 5 dimensões do QVA-r dos cursos de Odontologia e Medicina

\begin{tabular}{ccccccc}
\hline & \multicolumn{2}{c}{ Soma dos pontos } & \multicolumn{2}{c}{ Média ( \pm DP) } & \multicolumn{2}{c}{ Mediana } \\
Dimensões & Odontologia & Medicina & Odontologia & Medicina & Odontologia & Medicina \\
\hline Pessoal & 52,9 & 52 & $3,9 \pm 2,2 \mathrm{~A}$ & $3,9 \pm 2,4 \mathrm{~A}$ & 4,6 & 4,3 \\
Interpessoal & 44,1 & 44,8 & $3,7 \pm 0,4 \mathrm{~A}$ & $3,8 \pm 0,4 \mathrm{~A}$ & 3,7 & 3,8 \\
Carreira & 45,8 & 49,6 & $3,8 \pm 0,4 \mathrm{~B}$ & $4,1 \pm 0,6 \mathrm{~A}$ & 3,9 & 4,3 \\
Estudo & 34,8 & 31,6 & $3,9 \pm 0,7 \mathrm{~A}$ & $3,6 \pm 0,5 \mathrm{~B}$ & 4,1 & 3,6 \\
Institucional & 28,9 & 25,9 & $4,1 \pm 0,2 \mathrm{~A}$ & $3,7 \pm 0,2 \mathrm{~B}$ & 4,1 & 3,7 \\
\hline QVAr & 222,3 & 220,4 & $3,7 \pm 0,7 \mathrm{~A}$ & $3,7 \pm 0,6 \mathrm{~A}$ & 3,9 & 3,8 \\
\hline
\end{tabular}

Médias seguidas de letras distintas, para cada dimensão e instrumento completo, diferem entre si, considerando os cursos de matrícula (Teste t de Student)

A participação em programa de iniciação científica durante a graduação contribui para a formação profissional e deve ser valorizada pelas IES, favorecendo uma completa e crítica formação profissional $^{3,6,13,14}$. A possibilidade de ingressar em programas de IC é uma das principais oportunidades que as IES podem oferecer aos seus estudantes para integração ao ambiente acadêmico e leva à premissa de que a criação de programas institucionais de fato oferece um diferencial $^{15}$. Na Inglaterra, a participação precoce em programas de pesquisa favoreceu a tomada de decisão dos graduandos em Medicina para realização do estágio intercalado de pesquisa, ano "sabático" voluntário durante a formação médica para a realização de pesquisa ${ }^{14}$.

Na IES avaliada, estudo anterior ${ }^{5}$ constatou que em suas 4 primeiras edições do PIC o rendimento acadêmico dos estudantes vinculados ao programa foi diferenciado em relação àqueles que nunca participaram, porém verificou-se diferenças entre os 
cursos: para os alunos do curso de Odontologia, houve um significativo aumento $\mathrm{CR}$, calculado antes e depois do PIC, e para os alunos do curso de Medicina houve significativa diminuição deste coeficiente no mesmo período.

Buscando compreender esse achado, a vivência acadêmica dos alunos PIC foi avaliada considerandose as dimensões e a pontuação geral do QVA-r, comparando-se os grupos por curso de matrícula. $\mathrm{O}$ instrumento em questão permite a compreensão de aspectos pessoais, contextuais e vocacionais das vivências acadêmicas ${ }^{9}$ além de ter sido utilizado em diversos estudos de temática similar ${ }^{10,11}$ e apresenta níveis satisfatórios de consistência interna, em todas as dimensões de avaliação ${ }^{16}$.

Não se verificou diferença entre os cursos, considerando as dimensões pessoal e interpessoal. $\mathrm{Na}$ primeira encontram-se aspectos relacionados ao equilíbrio emocional, bem-estar físico, psicológico, otimismo e nas tomadas de decisões. A segunda avalia a capacidade de fazer e concretizar novas amizades, o que inclui a facilidade de estabelecer e concretizar laços de confiança. Com esses achados pode-se inferir que, de modo geral, a vida pessoal e a relação com os colegas da instituição não são fatores que possam estar relacionados à diferença encontrada entre os cursos, quanto ao rendimento acadêmico pós PIC.

$\mathrm{Na}$ dimensão estudo, a pontuação para os estudantes de Odontologia foi significativamente maior do que para Medicina. Nesta dimensão pode-se inferir que os acadêmicos de Odontologia consigam administrar melhor o tempo, encontrando maneiras para concretizar os estudos e se preparar para os testes aplicados pela instituição, sugerindo-se que possuam a capacidade de autorregulação da aprendizagem, processo consciente e voluntário que envolve planejamento, execução e avaliação da aprendizagem de forma cíclica. Neste sentido, o estudante exerce, com base em suas metas pessoais e padrões de conduta (comportamental, pensamentos e sentimentos), a gerência dos seus estudos ${ }^{17}$, sendo necessária, no entanto, uma investigação mais específica para validação desta hipótese.

Em relação à dimensão institucional, cuja pontuação também foi maior para os alunos do curso de Odontologia, aborda-se a afinidade com a instituição em que estão matriculados, a vontade de permanecer e a satisfação com a infraestrutura oferecida. A partir desta informação é possível sugerir que os estudantes de Odontologia possuem um maior envolvimento com a instituição, que é, por sua vez, preditor para a aprendizagem, para o desenvolvimento e para a permanência no ensino superior $^{18}$, sendo provavelmente um fator que influiu positivamente no CR da Odontologia.

O que pode ser relatado também em relação às dimensões estudo e institucional é que a atual característica do mercado de trabalho em Odontologia ${ }^{19}$, a competitividade nos concursos públicos, além dos conceitos obtidos no ENADE pelo curso da IES nos últimos anos, possam ter tido um efeito de responsabilização dos alunos, oportunizando a criação de uma rotina mais estruturada de estudos, no sentido de terem uma chance real de passar em concursos, além de manterem as notas da IES no patamar que as turmas anteriores atingiram. $\mathrm{O}$ resultado deste comportamento é o senso de pertencimento, uma ligação, muitas vezes expressa como um compromisso e/ou interesse comum, que serve para ligar o estudante à instituição, mesmo quando surgem desafios ${ }^{20}$.

Para os alunos do curso de Medicina, a dimensão carreira foi mais expressiva e refere-se à expectativas sobre a carreira, satisfação e a competência que o curso impõe, o que corrobora com $\mathrm{o}$ verificado em estudo anterior ${ }^{21}$. Os estudantes do curso de Medicina demonstraram ter maior confiança 
quanto à aceitação no mercado de trabalho e às perspectivas de remuneração com o curso de formação, enquanto os de Odontologia obtiveram menor pontuação na dimensão carreira, provavelmente relacionada à insegurança em relação a profissão e perspectivas de carreira, visto que o dentista já formado relata dificuldades para se colocar no mercado de trabalho ${ }^{19}$, que apresenta característica de saturação e competitividade, com concentração de profissionais nas regiões mais desenvolvidas e ricas do país $^{22}$. Em contraponto, vale ressaltar que a inclusão da saúde bucal na Estratégia Saúde da Família $^{23}$ ampliou as perspectivas de mercado de trabalho para o cirurgião-dentista, despontando o serviço público como alternativa promissora $^{24} \mathrm{e}$ almejada por muitos ${ }^{25}$, o que leva à necessidade da formação comprometida com a Saúde Pública/Saúde Coletiva para que possam atuar nos moldes desta estratégia.

Vale ressaltar que a carga horária $(\mathrm{CH})$ mínima para cursos de Medicina, conforme as Diretrizes Curriculares Nacionais ${ }^{26}$ é de 7.200 horas, enquanto que a $\mathrm{CH}$ do curso de Medicina da IES da presente pesquisa é extensa, apresentando 9.057 horas, o que pode ter influenciado nos resultados, pois os estudantes podem ter menos tempo livre para conciliar pesquisa e estudo. $\mathrm{O}$ projeto pedagógico do referido curso está sendo reavaliado com vistas à implementação de períodos livres para estudo, os quais têm se configurado como um indicador de qualidade das escolas médicas no âmbito internacional e nacional ${ }^{27,28}$. A média de horas/aula por semana é de 38h/semana no curso, no Brasil em média tem-se 30h/semana e, em currículos internacionais a carga horária é sempre inferior e demonstra que a presença de tempo livre oportuniza a realização de pesquisa ${ }^{14}$.

Os estudantes de Odontologia demonstraram, pelos resultados, um interesse maior pela instituição e maior conhecimento sobre a infraestrutura e o que ela pode proporcionar. Uma possível explicação para esse achado refere-se ao fato de que este curso, cuja primeira turma iniciou em 2004, já tem uma história consolidada. O curso de Medicina é mais recente, formou sua primeira turma em 2018 e como todos os cursos em formação, ao final deste primeiro ciclo, reformulará seu Projeto Pedagógico, inserindo mais períodos livres para que os alunos possam se dedicar a atividades extras como IC, Ligas Acadêmicas, atividades culturais e esportivas.

\section{CONCLUSÃO}

Vivências acadêmicas diferenciadas entre os cursos podem ter influenciado o antagônico impacto do PIC no CR dos estudantes de Odontologia e Medicina. A vivência acadêmica, especialmente considerando-se as dimensões carreira, estudo e institucional, mostraram-se diferentes para os alunos dos cursos, com vivências mais positivas para a Medicina, na primeira das dimensões citadas e mais positivas para os alunos da Odontologia nas duas outras.

Apoio financeiro: Bolsa do programa de iniciação cientifica institucional São Leopoldo Mandic (001-AIE-2017)

\section{ABSTRACT \\ Scientific mentorship, academic experiences and undergraduate performance of Dentistry and Medicine students}

The Scientific Mentorship Program (SMP) seeks to foster vocational aims and encourage the development of scientific thought in undergraduate students, and participation in the program has been found to have a positive impact on the performance coefficient (PC) of Dentistry students, but not in students from the Medicine course. The present observational study was 
therefore conducted to identify the personal, contextual and vocational aspects of the academic experiences of Medicine and Dentistry students, which may have influenced this finding. A total of 123 students participated in the study, of whom 74 $(60.2 \%)$ were from the Dentistry (D) course and 49 (39.9\%) from the Medicine (M) course. The short version of the Student Academic Experience Questionnaire (or QVA-r) was applied (54 questions/Likert Scale responses). The data were tabulated and submitted to statistical analysis using the Student's t-test $(\alpha=5 \%)$. The main results revealed that the career, study and institutional dimensions differed between the groups, with higher mean scores obtained by the students of the Medicine course in the career dimension $(\mathrm{M}=4.1 \pm 0.6 \mathrm{a} ; \mathrm{D}=3.8 \pm 0.4 \mathrm{~b})$. Higher scores were obtained for students from the Dentistry course in the study $(M=3.6 \pm 0.5 \quad b ; D=3.9 \pm 0.7 a)$ and institutional $(M=3.7 \pm 0.2 \quad b ; \quad D=4.1 \pm 0.2 a)$ dimensions. There was no difference between the courses in the personal and interpersonal dimensions, or in the overall QVAr score. It was concluded that different academic experiences may have influenced the contrasting impact of the SMP on the PC of the Dentistry and Medicine students.

Descriptors: Scientific Research. Academic Achievement. Professional Education.

\section{REFERÊNCIAS}

1. Massi LQ, Queiroz SLL. Studies on undergraduate research in Brazil: a review. Rev Eletr Cad Pesqui. 2010;40(139):173-97.

2. Gorgens JB. Avaliação da produção científica dos egressos, bolsistas e não bolsistas de iniciação científica, do curso de medicina da Universidade Federal de Minas Gerais, de 1994 a 1999, pelo currículo Lattes (tese) Belo Horizonte: Universidade Federal de Minas Gerais; 2007.

3. Machineski RS, Machado ACTA, Silva RTM.
A importância do estágio e do programa de iniciação científica na formação profissional e científica. Enciclop Biosf. 2011;7(13):156274.

4. Nardini EF, Turssi CP, Fonseca Silva AS, Flório FM. Política de estímulo à iniciação científica: impacto no coeficiente de rendimento de graduandos em Odontologia. Rev ABENO. 2019;19(1):33-9.

5. Nardini EF, Flório FM. Acompanhamento de ingressantes e egressos do programa de iniciação científica da Faculdade São Leopoldo Mandic. In: Seminário de Iniciação Científica; 2017 out 3; Campinas.

6. Cunha SM, Carrilho DM. O processo de adaptação ao ensino superior e o rendimento acadêmico. Psicol Esc Educ. 2005;9(2):21524.

7. Soares AB, Francischetto V, Dutra BM, Miranda JM, Nogueira C, Leme VR, Araújo AM, Almeida LS. O impacto das expectativas na adaptação acadêmica dos estudantes no Ensino Superior. PsicoUSF. 2014; 19(1):4960.

8. Monteiro MC, Soares AB. Adaptação acadêmica de estudantes cotistas e não cotistas. Rev Bras Orient Profiss. 2018 19(1):51-60.

9. Igue ÉA, Bariani ICD, Milanesi PVB. Vivência acadêmica e expectativas de universitários ingressantes e concluintes. PsicoUSF. 2008; 13(2):155-64.

10. Noronha APP, Martins DDF, Gurgel MGDA, Ambiel RAM. Estudo correlacional entre interesses profissionais e vivências acadêmicas no ensino superior. Psicol Esc Educ. 2009; 13(1):143-54.

11. Soares AB, dos Santos Mello TV, Baldez MDOM. Vivências acadêmicas em estudantes 
universitários do Estado do Rio de Janeiro. Interação Psicol. 2011;15(1):59-69.

12. Granado JIF, Santos AAA, Almeida LS, Soares AP, Guisande MA. Integração académica de estudantes universitários: contributos para a adaptação e validação do QVA-r no Brasil. Psicol Educ. 2005;4(2):3141.

13. Bastos F, Martins F, Alves M, Terra M, Lemos CS. A Importância da Iniciação Científica para os alunos de graduação em Biomedicina. Rev Eletr Novo Enfoque. 2010;11(11):61-6.

14. Boyle SE, Cotton SC, Myint PK, Hold GL. The influence of early research experience in medical school on the decision to intercalate and future career in clinical academia: a questionnaire study. BMC Med Educ. 2017;17(1):245.

15. Chisini LA, Silva HG, Nóbrega KHS, Conde MCM, Corrêa MB, Demarco FF. Análise descritiva dos trabalhos de conclusão de curso da Faculdade de Odontologia. Rev ABENO. 2017;17(1):8-15.

16. Almeida LS, Ferreira JÁ, Soares AP. Questionário de Vivências Académicas (QVA e QVA-r). In: MM Gonçalves, MR Simões, LS Almeida, Machado C. Avaliação psicológica: Instrumentos validados para a população portuguesa. 2003.

17. Polydoro SAJ, Azzi RG Autorregulação da aprendizagem na perspectiva da teoria sociocognitiva: introduzindo modelos de investigação e intervenção. Psic Educ. 2009. 29(2):75-94.

18. Fior CA, Mercuri E. Envolvimento acadêmico no ensino superior e características do estudante. Rev Bras Orient Profiss. 2018;19(1):85-95.
19. Ferreira NP, Ferreira AP, Freire MCM. Mercado de trabalho na odontologia: contextualização e perspectivas. Rev Odontol UNESP. 2013;42(4):304-9.

20. Tinto V. Through the eyes of students. J Coll Student Ret. 2017;19(3):254-69.

21. dos Anjos DRL, Aguilar-da-Silva RH. Questionário de Vivências Acadêmicas (QVA-R): avaliação de estudantes de medicina em um curso com currículo inovador. Avaliação. 2017;22(1):105-23.

22. San Martin A, Chisini L, Martelli S, Sartori L, Ramos E, Demarco F. Distribution of dental schools and dentists in Brazil: an overview of the labor market. Rev ABENO. 2018;18(1):63-73.

23. Brasil. Ministério da Saúde. Portaria n. 267/GM, de 06 março de 2001. Define as Diretrizes e Normas da inclusão das ações de saúde bucal no PSF. Diário Oficial da República Federativa do Brasil, Brasília (DF); 2001 mar 7; Seção 1:67. [Acesso em 21/08/2018]. Disponível em: https://www. nescon.medicina.ufmg.br/biblioteca/

24. Silveira Filho AD. A Saúde Bucal no PSF: o desafio de mudar a prática. Rev Saúde Família. 2002; 6:36-43.

25. Silva CV, Spiger V, Amante CJ. Perfil e expectativas profissionais de concluintes do curso de graduação em Odontologia da Universidade Federal de Santa Catarina. Rev ABENO. 2018; 18(3):35-42.

26. Conselho Nacional de Educação. Parecer CNE/CES no 116/2014, de 20 de junho de 2014: diretrizes curriculares nacionais do curso de graduação em medicina. Brasília: Ministério da Educação; 2014.

27. Hunt D, Migdal M, Waechter DM, Barzansky B, Sabalis RF. The variables that lead to 
severe action decisions by the liaison Flávia Martão Flório

Committee on Medical Education. Acad Med. email: flaviaflorio@yahoo.com

2016;91(1):87-93.

flavia.floio@slmandic.edu.br

28. SAEME. Sistema de Acreditação de Escolas Rua José Rocha Junqueira, 13

Médicas. Instrumento de Avaliação do 13045-755 Campinas/SP

SAEME; 30 jun. 2015. [Acesso em

21/08/2018]. Disponível em: http://saeme.

org.br/images/saeme_instrumento_final.pdf.

\section{Correspondência para:}

